\title{
Alternating Stacking of Nanocrystals and Nanofibers into Ultra-Strong Chiral Biocomposite Laminates
}

Xiaofang Zhang, ${ }^{1,2} \S$ Rui Xiong, ${ }^{1}$ Saewon Kang, ${ }^{1}$ Yingkui Yang, ${ }^{* 2}$ Vladimir V.

$T_{\text {sukruk }}^{* 1}$

${ }^{1}$ School of Materials Science and Engineering, Georgia Institute of Technology, Atlanta, GA 30332-0245, USA

${ }^{2}$ Key Laboratory of Catalysis and Energy Materials Chemistry of Ministry of Education \& Hubei Key Laboratory of Catalysis and Materials Science, South-Central University for Nationalities, Wuhan 430074, China

Xiaofang Zhang and Rui Xiong contributed equally to this work.

*Corresponding authors: ykyang@mail.scuec.edu.cn; vladimir@mse.gatech.edu; 

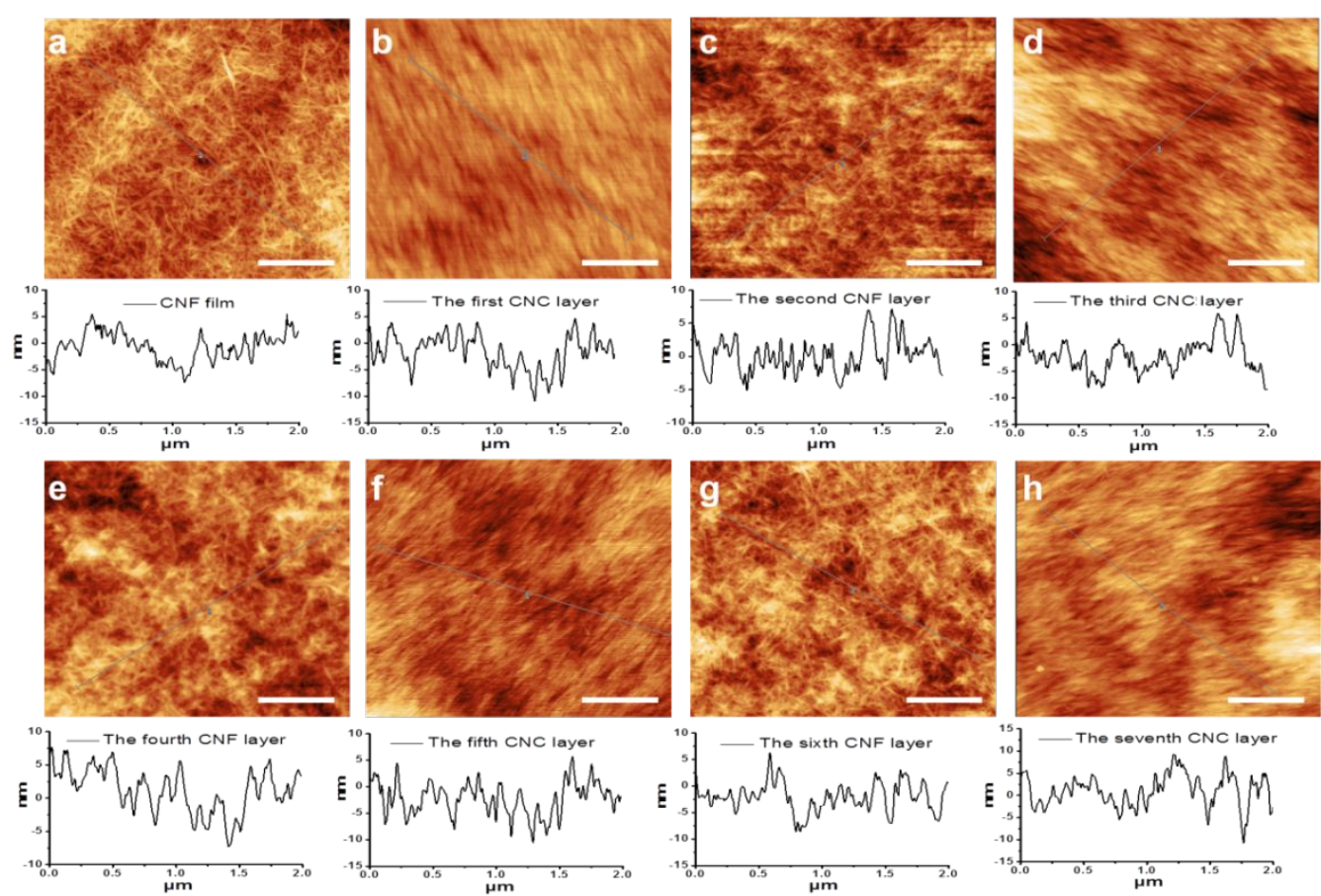

Figure S1. The surface topology AFM images (top view) of (a) CNF, (b) CNC, (c) C1F1, (d) C2F1, (e) C2F2, (f) C3F2, (g) C3F3, and (h) C4F3 films. Scale bar: 500 nm. 


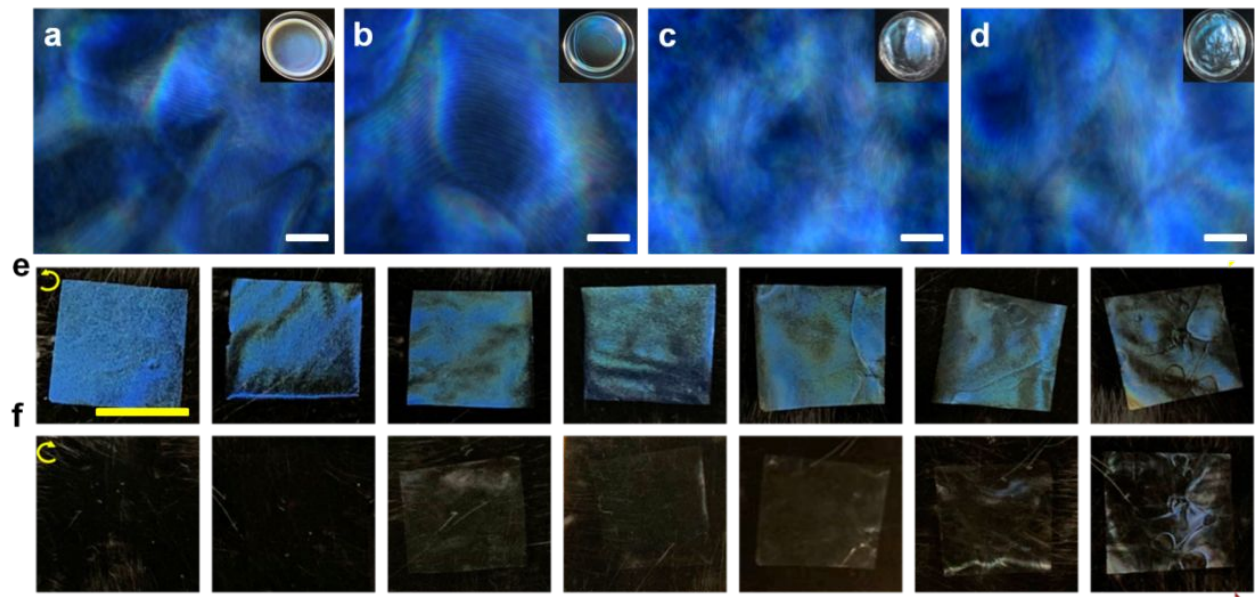

Figure S2. POM images taken with crossed polarizers for CNC (a), C1F1 (b), C2F2 (c), and C3F3 (d) films. Scale bar: $10 \mu \mathrm{m}$. Insets: photos of CNC, C1F1, C2F2, and C3F3 films; Photos of all the films taken under left-handed (e) and right-handed (f) circular polarizer. From left to right: CNC, C1F1, C2F1, C2F2, C3F2, C3F3 and C4F3 films, successively. Scale bar: $1 \mathrm{~cm}$. 

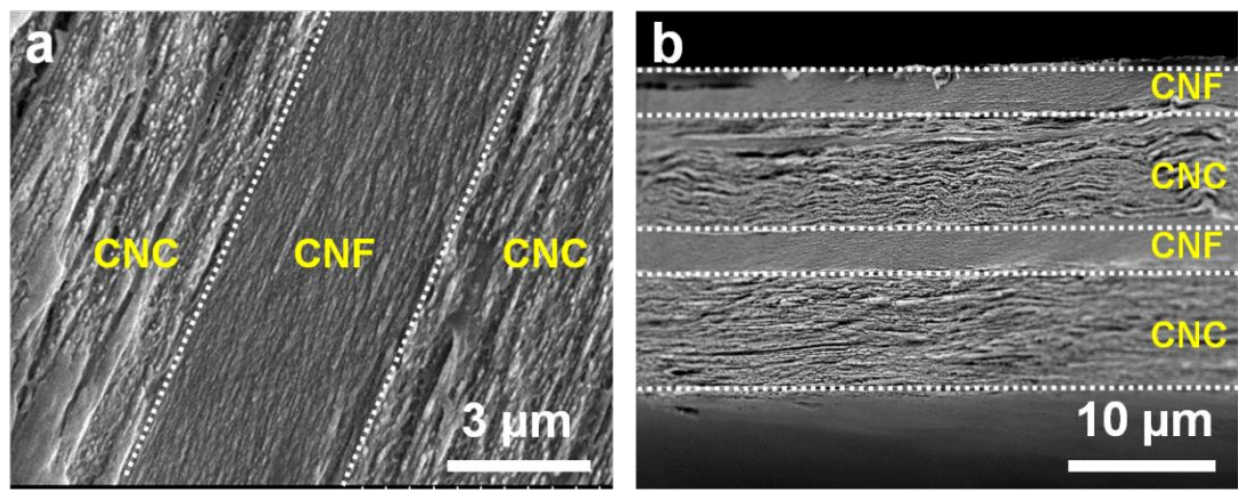

Figure S3. Cross section SEM images of C2F1 three-layer (a) and C2F2 four-layer (b) films. 


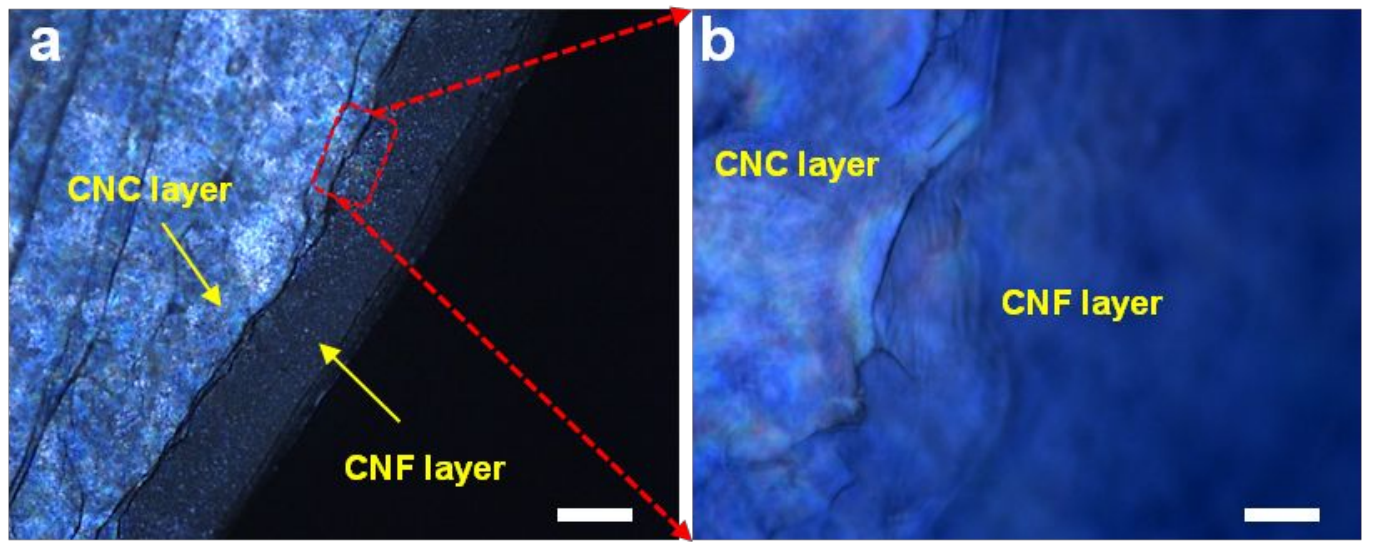

Figure S4. Cross-section POM images of C1F1 film after tearing in ambient condition taken with crossed polarizer, Scale bar: $100 \mu \mathrm{m}$ (a) and $20 \mu \mathrm{m}$ (b). 

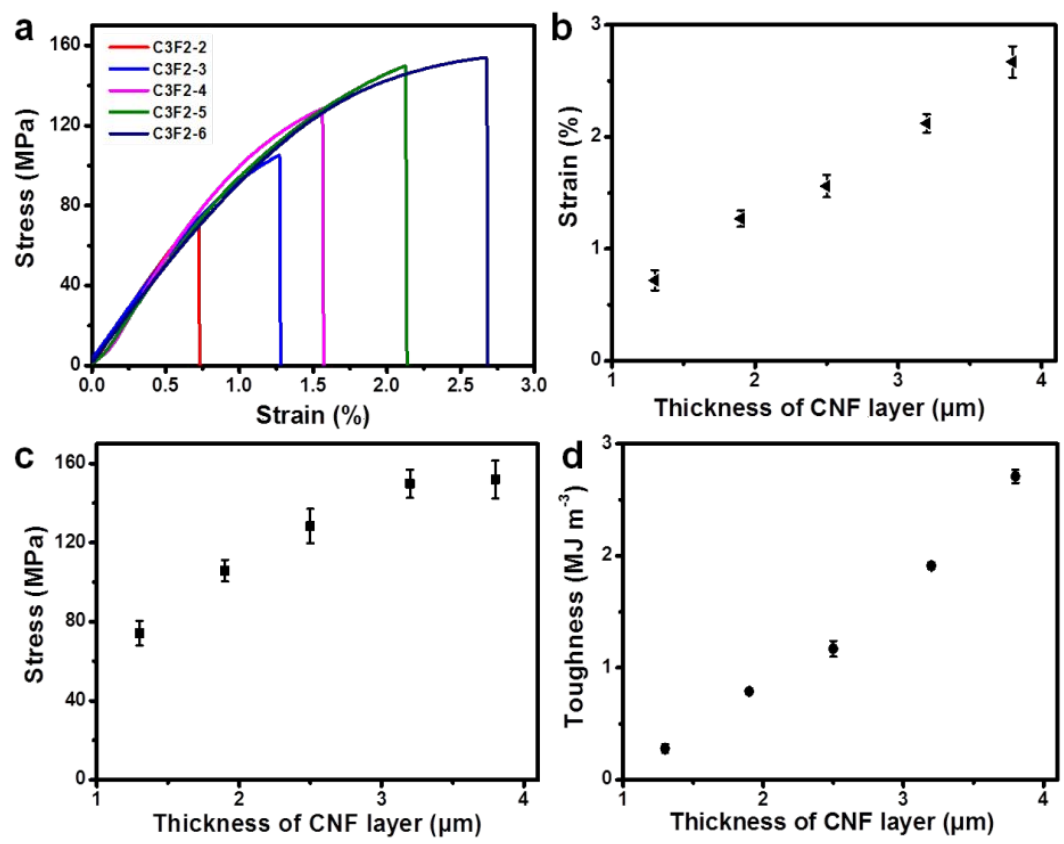

Figure S5. (a) Stress-strain curves for C3F2-n, n (ml) refers to the volume of CNF suspension used for layer-by-layer drop casting. The (b) elongation at break, (c) tensile strength, and (d) toughness as a function of the thickness of CNF layer in 5-layered C3F2 films. 
A mixed suspension of CNCs and CNFs was subjected to an evaporation-induced self-assembly to yield the homogeneous $\mathrm{CNC} / \mathrm{CNF}$ film with the same $\mathrm{CNC} / \mathrm{CNF}$ mass ratio as the 5-layered $\mathrm{C} 3 \mathrm{~F} 2$ film. The $\mathrm{CNC} / \mathrm{CNF}$ blend film is colorless (Figure S6a), indicating the collapse of inherently ordered orientation of CNCs (Figure S6b, c). It becauses the presence of long CNFs interpenetrated with needle-like CNCs for suppressing the helical self-assembly. The mechanical strength of the $\mathrm{CNC} / \mathrm{CNF}$ blend film is inferior to that of the multilayered C3F2 film (Figure S6c), possibly due to the relatively weak interfacial interaction and low load transfer capability in the $\mathrm{CNC} / \mathrm{CNF}$ blending film. While $\mathrm{CNC} / \mathrm{CNF}$ blend film and $\mathrm{C} 3 \mathrm{~F} 2$ film have a similar Young's modulus (Figure S6d), resulted from their identical CNC/CNF content.
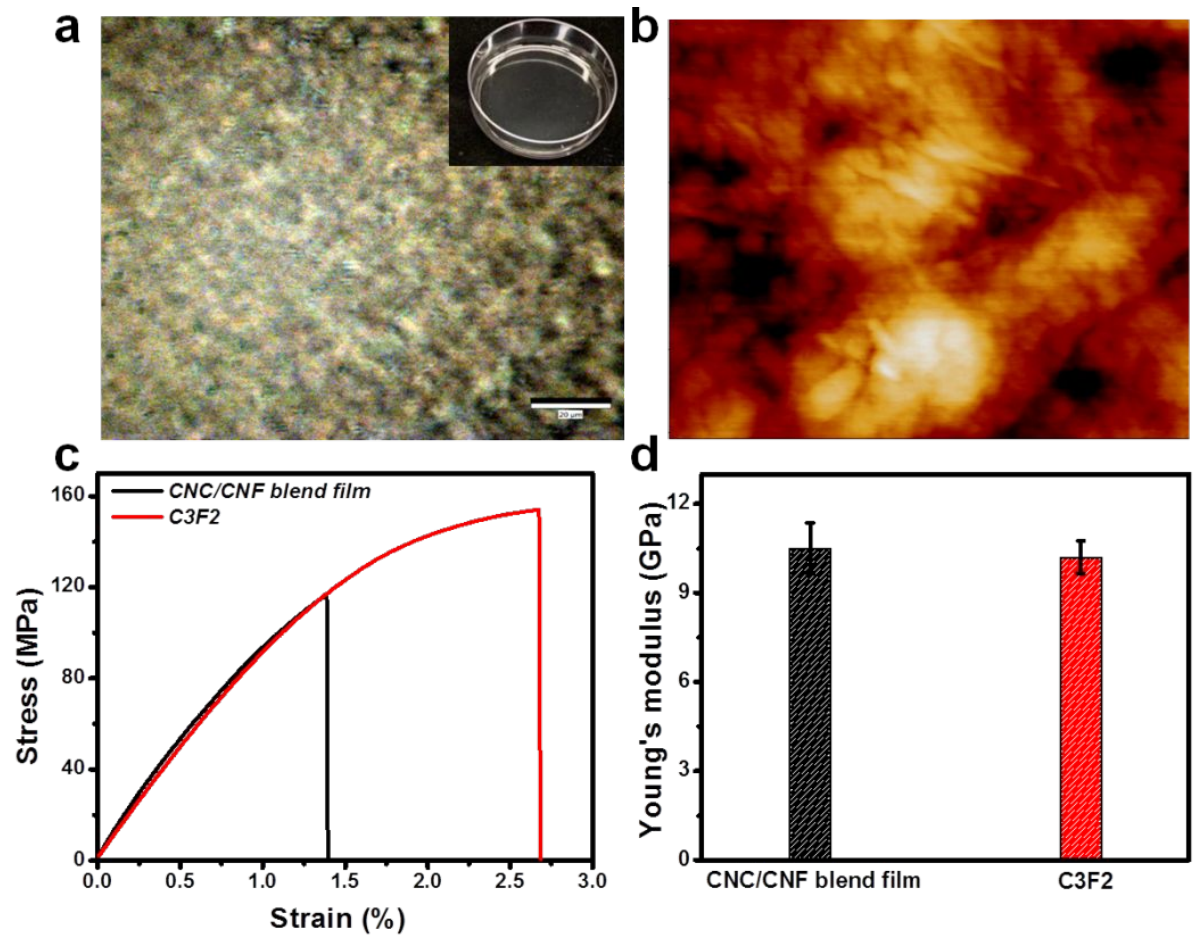

Figure S6. (a) $\mathrm{POM}$ image of $\mathrm{CNC} / \mathrm{CNF}$ blend film taken with crossed polarizer, inset is the Photo of $\mathrm{CNC} / \mathrm{CNF}$ blend film; (b) AFM image of $\mathrm{CNC} / \mathrm{CNF}$ blend film; (c) Stress-strain curves of CNC/CNF blending and C3F2 films; (d) Comparing the Young's modulus of CNC/CNF blend films and layered C3F2 films. 
Table S1. Component, thickness and mechanical properties of all the films studied in this work. $\left(\mathrm{C}_{\mathrm{c}}\right.$ and $\mathrm{C}_{\mathrm{f}}$ present $\mathrm{CNC}$ and $\mathrm{CNF}$ content, respectively; $\mathrm{BF}_{\mathrm{C}-\mathrm{F}}$ presents the CNC-CNF blend film)

\begin{tabular}{|c|c|c|c|c|c|c|c|c|c|c|}
\hline Materials & CNF & $\mathrm{CNC}$ & C1F1 & C2F1 & $\mathrm{C} 2 \mathrm{~F} 2$ & $\mathrm{C} 3 \mathrm{~F} 2$ & C3F3 & C4F3 & F3C2 & $\mathbf{B F}_{\mathrm{C}-\mathrm{F}}$ \\
\hline $\mathrm{C}_{\mathrm{c}} / \%$ & I & 100 & 69.8 & 82.2 & 69.8 & 77.6 & 69.8 & 75.5 & 61 & 77.6 \\
\hline $\mathrm{C}_{\mathrm{f}} / \%$ & 100 & I & 30.2 & 17.8 & 30.2 & 22.4 & 30.2 & 24.5 & 39 & 22.4 \\
\hline $\begin{array}{c}\text { Calculated } \\
\text { thickness }(\mu \mathrm{m})\end{array}$ & l & / & 10.5 & 17.2 & 21 & 27.7 & 31.5 & 38.2 & l & l \\
\hline Measured & 3.8 & 6.7 & 10.7 & 17.6 & 22 & 28 & 31 & 37 & I & / \\
\hline thickness $(\mu \mathrm{m})$ & \pm 1.53 & \pm 2.5 & \pm 2.32 & \pm 2.16 & \pm 1.41 & \pm 1.63 & \pm 1.15 & \pm 1.41 & & \\
\hline Elongation to & 3.3 & 0.35 & 1.30 & 1.37 & 1.42 & 2.68 & 2.31 & 1.89 & 2.81 & 1.35 \\
\hline break $\left(\varepsilon_{\mathrm{f}}\right) / \%$ & \pm 0.13 & \pm 0.05 & \pm 0.07 & \pm 0.11 & \pm 0.09 & \pm 0.14 & \pm 0.49 & \pm 0.13 & \pm 0.21 & \pm 0.06 \\
\hline Strength & 170 & 46.2 & 87.6 & 115.7 & 120.9 & 151.6 & 119.7 & 114.6 & 161 & 114.6 \\
\hline$\left(\sigma_{\mathrm{f}}\right) / \mathrm{MPa}$ & \pm 15.7 & \pm 4.8 & \pm 4.7 & \pm 5.4 & \pm 4.2 & \pm 9.4 & \pm 8.0 & \pm 6.1 & \pm 9.5 & \pm 2.26 \\
\hline Young's & 8.6 & 10.8 & 10.0 & 10.5 & 10.1 & 10.2 & 9.9 & 10.1 & 10.1 & 10.5 \\
\hline (E)/GPa & \pm 0.25 & \pm 0.70 & \pm 1.23 & \pm 0.51 & \pm 1.11 & \pm 0.55 & \pm 0.35 & \pm 0.49 & \pm 0.26 & \pm 0.86 \\
\hline Toughness /MJ & 3.8 & 0.077 & 0.506 & 0.896 & 1.039 & 2.715 & 2.133 & 3.365 & 2.851 & $0.899 \pm$ \\
\hline $\mathrm{m}^{-3}$ & \pm 1.53 & \pm 0.012 & \pm 0.022 & \pm 0.023 & \pm 0.023 & \pm 0.065 & \pm 0.018 & \pm 0.071 & \pm 0.021 & 0.027 \\
\hline
\end{tabular}


Table S2. Comparison in tensile strength and toughness between the stacked $\mathrm{CNC} / \mathrm{CNF}$ films in this study and the previously reported CNC-based chiral films.

\begin{tabular}{|c|c|c|c|}
\hline Material & $\begin{array}{c}\text { Tensile strength } \\
\text { (MPa) }\end{array}$ & $\begin{array}{c}\text { Toughness } \\
\left(\mathrm{MJ} \mathrm{m}^{-3}\right)\end{array}$ & Ref \\
\hline CNC chiral film & 33 & 0.06 & 1 \\
\hline CNC chiral film & 33 & 0.08 & 2 \\
\hline CNC chiral film & 26 & 0.04 & 3 \\
\hline CNC chiral film & 14 & 0.02 & 4 \\
\hline CNC chiral film & 35 & 0.12 & 5 \\
\hline $\mathrm{CNC} / \mathrm{CNF}$ & 37 & 0.26 & 4 \\
\hline $\mathrm{CNC} / \mathrm{T}-\mathrm{CNC}$ & 74 & 0.23 & 1 \\
\hline CNC/DMAPS & 63 & 0.25 & 6 \\
\hline $\mathrm{CNC} / \mathrm{PEG}$ & 48 & 0.43 & 7 \\
\hline $\mathrm{CNC} / \mathrm{PVA}$ & 67 & 0.27 & 8 \\
\hline CNC/Latex NPs & 29 & 0.12 & 2 \\
\hline $\mathrm{CNC} /$ Ionic liquid & 15 & 0.07 & 3 \\
\hline CNC/Glycerol & 40 & 0.40 & 9 \\
\hline $\mathrm{CNC} / \mathrm{PEG}$ & 29 & 0.25 & 5 \\
\hline $\mathrm{CNC} / \mathrm{WPU}$ & 42 & 0.35 & 10 \\
\hline CNC/EGUPy2 & 27 & 0.12 & 11 \\
\hline C3F2 & 151.6 & 2.72 & This work \\
\hline
\end{tabular}




\section{References}

1. Natarajan, B.; Krishnamurthy, A.; Qin, X.; Emiroglu, C. D.; Forster, A.; Foster, E. J.; Weder, C.; Fox, D. M.; Keten, S.; Obrzut, J.; Gilman, J. W., Binary Cellulose Nanocrystal Blends for Bioinspired Damage Tolerant Photonic Films. $A d v$. Funct.Mater. 2018, 28, 1800032.

2. Vollick, B.; Kuo, P.-Y.; Thérien-Aubin, H.; Yan, N.; Kumacheva, E., Composite Cholesteric Nanocellulose Films with Enhanced Mechanical Properties. Chem. Mater. 2017, 29, 789-795.

3. Liu, P.; Guo, X.; Nan, F.; Duan, Y.; Zhang, J., Modifying Mechanical, Optical Properties and Thermal Processability of Iridescent Cellulose Nanocrystal Films Using Ionic Liquid. ACS Appl. Mater. Interfaces 2017, 9, 3085-3092.

4. Xiong, R.; Han, Y.; Wang, Y.; Zhang, W.; Zhang, X.; Lu, C., Flexible, Highly Transparent and Iridescent All-Cellulose Hybrid Nanopaper with Enhanced Mechanical Strength and Writable Surface. Carbohydr. Polym. 2014, 113, 264-71.

5. Bardet, R.; Belgacem, N.; Bras, J., Flexibility and Color Monitoring of Cellulose Nanocrystal Iridescent Solid Films Using Anionic or Neutral Polymers. ACS Appl.Mater. Interfaces 2015, 7, 4010-4018.

6. Guidetti, G.; Atifi, S.; Vignolini, S.; Hamad, W. Y., Flexible Photonic Cellulose Nanocrystal Films. Adv. Mater. 2016, 28, 10042-10047.

7. Yao, K.; Meng, Q.; Bulone, V.; Zhou, Q., Flexible and Responsive Chiral Nematic Cellulose Nanocrystal/Poly(ethylene Glycol) Composite Films with Uniform and Tunable Structural Color. Adv. Mater. 2017, 29, 1701323.

8. Wang, B.; Walther, A., Self-Assembled, Iridescent, Crustacean-Mimetic Nanocomposites with Tailored Periodicity and Layered Cuticular Structure. ACS Nano 2015, 9, 10637-10646.

9. Xu, M.; Li, W.; Ma, C.; Yu, H.; Wu, Y.; Wang, Y.; Chen, Z.; Li, J.; Liu, S., Multifunctional Chiral Nematic Cellulose Nanocrystals/Glycerol Structural Colored Nanocomposites for Intelligent Responsive Films, Photonic Inks and Iridescent Coatings. J. Mater. Chem. C 2018, 6, 5391-5400.

10. Wan, H.; Li, X.; Zhang, L.; Li, X.; Liu, P.; Jiang, Z.; Yu, Z. Z., Rapidly Responsive and Flexible Chiral Nematic Cellulose Nanocrystal Composites as Multifunctional Rewritable Photonic Papers with Eco-Friendly Inks. ACS Appl. Mater. Interfaces 2018, 10, 5918-5925.

11. Fernandes, S. N.; Almeida, P. L.; Monge, N.; Aguirre, L. E.; Reis, D.; de Oliveira, C. L.; Neto, A. M.; Pieranski, P.; Godinho, M. H., Mind the Microgap in Iridescent Cellulose Nanocrystal Films. Adv. Mater. 2017, 29. 1603560. 